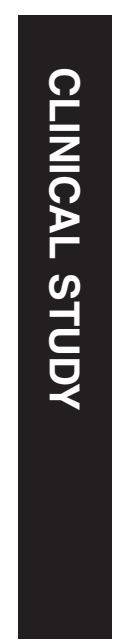

${ }^{1}$ The Royal Eye Hospital, Central Manchester University Hospitals NHS Foundation Trust, Manchester, UK

${ }^{2}$ Institute of Human Development, Faculty of Medical and Human Sciences, University of Manchester, Manchester, UK

${ }^{3}$ Manchester Vision Regeneration Lab at Manchester Royal Eye Hospital and NIHR/ Wellcome Trust Manchester CRF, Central Manchester University Hospital NHS Foundation Trust, Manchester, UK

Correspondence: NP Jones, FRCOphth, Consultant

Ophthalmologist, The Royal Eye Hospital, Oxford Road, Manchester, M13 9W UK Tel: +44 (0)161276 5628; Fax: +44 (0)1612726618. E-mail: Nicholas.jones@ cmft.nhs.uk

Received: 2 November 2016 Accepted in revised form: 15 April 2017 Published online: 2 June 2017

\title{
Ultra-widefield fundus fluorescein angiography in the diagnosis and management of retinal vasculitis
}

\begin{abstract}
Purpose To quantify the additional information provided by ultra-widefield fluorescein angiography, compared with 7-field standard imaging, in patients with retinal vasculitis (RV).

Patients and methods Retrospective case series of 106 patients.

Results Retinal vascular pathology was identified by UWF FFA, but not by standard ETDRS 7-field area, in 62 patients $(58.5 \%)$ and in 79 eyes $(43.4 \%)$. The pathology included active RV (47 eyes, $25.8 \%$ of eyes); retinal ischaemia, or infarction (53 eyes, $29.1 \%$ ); and retinal neovascularization (7 eyes, $3.8 \%$ ). A change to management was made in 36 patients $(34 \%)$. Of these, $21(20 \%$ of all patients undergoing angiography) were made after the identification of retinal vascular pathology, which was found only on UWF FFA, outside the ETDRS area.

Conclusion Ultra-widefield fluorescein angiography has clear advantages over standard multi-field imaging. It is currently the standard method of assessment for RV in this centre.
\end{abstract} Eye (2017) 31, 1546-1549; doi:10.1038/eye.2017.93; published online 2 June 2017

\section{Introduction}

Retinal vasculitis (RV) is uncommon, but may be the primary focus of intraocular inflammation. A recent study from the Manchester Uveitis Clinic (MUC) found that in $4.5 \%$ of patients with intraocular inflammation, RV was the predominant feature. ${ }^{1}$ Occlusive disease in particular is sight-threatening and RV may require aggressive management including antimicrobial treatment for infective inflammation, or immunosuppression for non-infective disease. The ability to diagnose important manifestations of
NP Jones ${ }^{1,2}$, A Sala-Puigdollers ${ }^{1,3}$ and PE Stanga ${ }^{1,2,3}$
$R V$, including vascular wall staining, extravascular leakage, retinal ischaemia or infarction, and retinal neovascularization, is important in decision-making.

Standard multiple $30^{\circ}$ fundus fluorescein angiography (FFA) images (the mainstay of RV imaging for 4 decades) even if performed diligently in logical order, requires a high degree of mental manipulation by the observer to recreate a fundal picture of disease, and sequential and 'round the clock' narrow-field imaging to capture all clock hours of the fundus implies that no fields are simultaneous due to the time lapse between images. In addition, direct clinical observation in patients with uveitis is may be challenging because of media opacity or compromised pupils.

The introduction of widefield imaging using the Staurenghi contact and non-contact lenses in conjuntion with the Heidelberg Engineering SPECTRALIS system (Heidelberg Engineering GmbH, Robert-Koch-Straße 1, 69115 Heidelberg, Germany) ${ }^{2}$ and the Optos Ultra Wide-Field (UWF) (Optomap, Dunfirmline, UK) ${ }^{3}$ has facilitated the process of photography but most importantly that of image analysis and interpretation.

UWF FFA imaging technologies have removed some of these limitations, especially by allowing, for the first time, simultaneous and high-contrast angiographic images of all 360 degrees of the mid and peripheral retina. The use of UWF imaging confers the advantage of rapid pattern recognition, with potential resultant earlier diagnosis and prediction of disease progression. The confocal nature of Optos technology also permits the user to bypass some of the interference induced by cataract or vitreous opacities, and to image through small pupils, these being common components of uveitis. 
Optos imaging technology involves the use of a scanning laser ophthalmoscope of two wavelengths (green $532 \mathrm{~nm}$ and red $633 \mathrm{~nm}$ ) in combination with a large concave elliptical mirror, obtaining a retinal image field of $180-200^{\circ}$ ( $82 \%$ of the retina) and high-resolution images (up to 2000 by 2000 pixels) within 0.25 s. This study aims firstly to quantify the additional information provided by Optos UWF angiography in patients with RV in comparison with standard $30^{\circ}$ imaging, and secondly to quantify effects on patient management.

\section{Subjects and methods}

MUC maintains a prospectively filled database of all new patients attending, containing information on diagnosis. All patients with RV as the predominant manifestation of intraocular inflammation, were identified and the images of those who had undergone UWF FFA using the Optos 200Tx system were analysed. Criteria noted included active RV (retinal vessel wall staining with fluorescein leakage), vascular occlusion with retinal ischaemia or infarction, and retinal neovascularization. The images onscreen were overlaid with a scaled standard $7 \times 30^{\circ}$ field ETDRS pattern, as shown in Figure 1 to obtain a comparison between these methods. Retinal vascular pathology was recorded by one of us (NPJ) as being present either within the comparative ETDRS area, or only outside it. The sensitivity for manifestations of RV was compared between simulated ETDRS and UWF FFA. Subsequent management decisions were noted, and the impact of UWF FFA on those decisions was analysed.

\section{Results}

There were 148 patients on the MUC database exhibiting $\mathrm{RV}$ as a predominant feature of their intraocular

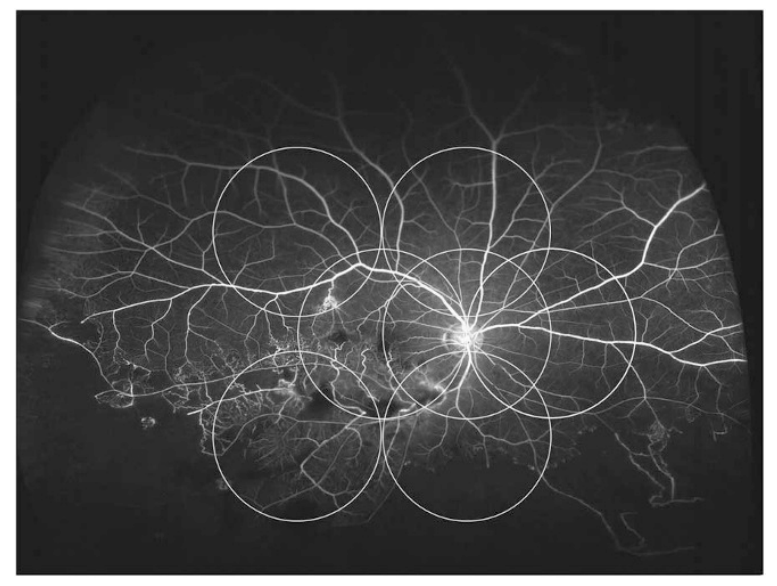

Figure 1 A standard ETDRS 7-field scaled grid superimposed on an ultra-widefield fluorescein angiogram. inflammation. Of these, 106 had undergone UWF FFA between February 2011 and June 2015. Bilateral RV was seen in $79(74.5 \%)$ of patients and unilateral in 27 (total 185 affected eyes). The underlying diagnoses are shown in Table 1; primary (idiopathic) RV was the most common (38 patients, 35.8\%), followed by sarcoidosis-associated $(19,17.9 \%)$ and Behçet's disease $(17,16 \%)$. Of the 27 patients with unilateral RV, the left eye was affected in 16 and the right in 11. This difference is not significant.

Medical or laser treatment had already been administered to 53 patients before their UWF FFA; of these, 46 patients (82 affected eyes) had received oral steroid and/or immunosuppression, and 18 patients (32 eyes) had undergone argon laser ablation to areas of retinal ischaemia.

All 106 patients (182 affected eyes) underwent UWF FFA. Examining the whole area of the UWF images, active RV was found in 133 eyes (73.1\%). Retinal vascular pathology was identified by UWF FFA, but not by standard ETDRS 7-field area, in 62 patients (58.5\%) and in 79 eyes (43.4\%). The pathology included active RV (47 eyes, $25.8 \%$ of eyes, Figure 2); retinal ischaemia or infarction (53 eyes, 29.1\%, Figure 3); and retinal neovascularization (7 eyes, $3.8 \%$, Figure 4 ).

Following UWF FFA, a decision to change management was made in 36 patients (34\%). In 11 cases, laser was applied to ischaemic retina; in 14 cases,

immunosuppression was either commenced or increased in dosage (7 azathioprine, 5 mycophenolate, and 2 ciclosporin); in 3, anti-tuberculosis treatment was commenced, and in 8, oral steroid was commenced or increased in dosage. Of these 36 management decisions, 21 (20\% of all patients undergoing angiography) were made after the identification of retinal vascular pathology, which was found only on UWF FFA, outside the ETDRS area.

Table 1 Diagnoses of patients with retinal vasculitis

\begin{tabular}{lc}
\hline Diagnosis & No. of patients \\
\hline Bacterial endophthalmitis & 1 \\
Behçet's disease & 17 \\
Birdshot retinochoroidopathy & 6 \\
Cytomegalovirus retinitis & 2 \\
IRVAN syndrome & 1 \\
Multiple sclerosis-associated & 3 \\
Necrotizing herpetic retinitis & 2 \\
Primary (idiopathic) retinal vasculitis & 38 \\
Sarcoidosis-associated & 19 \\
Susac syndrome & 1 \\
Syphilis-associated & 2 \\
Tuberculosis-associated & 14 \\
Total & 106
\end{tabular}

Abbreviation: IRVAN, idiopathic retinal vasculitis with aneurysms and neuroretinitis. 


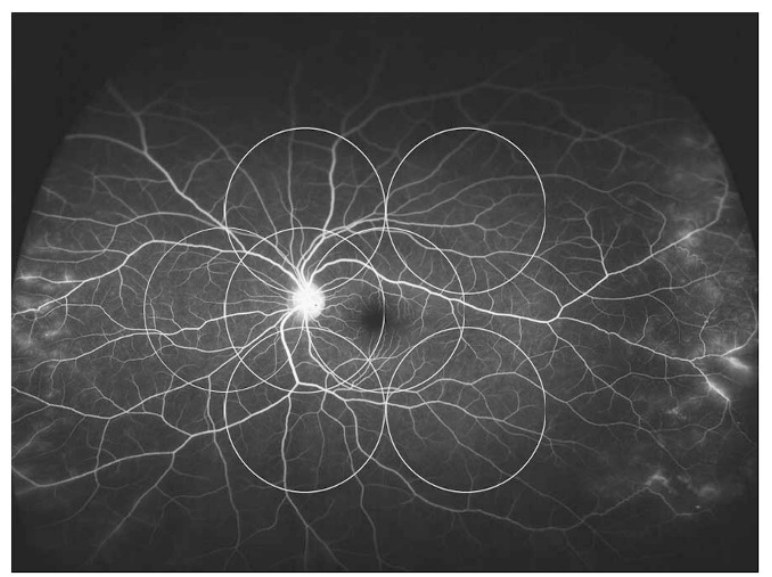

Figure 2 Active retinal vasculitis detected outside the ETDRS 7-field area.

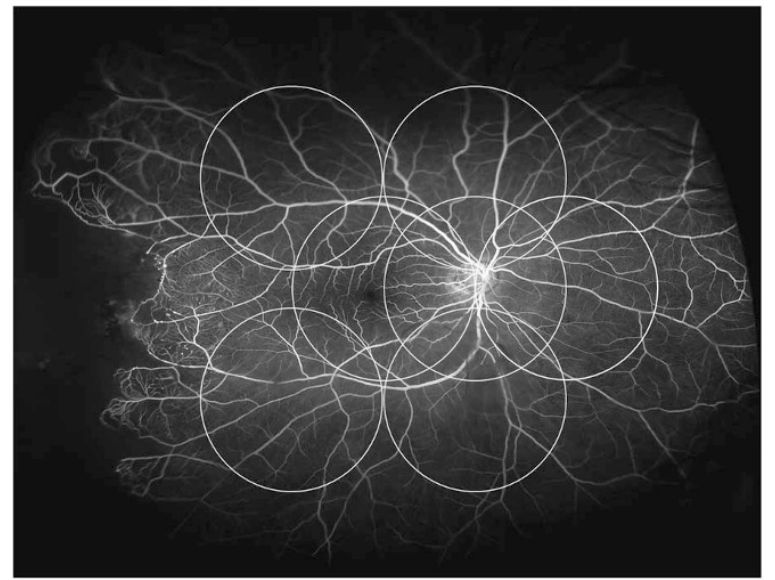

Figure 3 Retinal infarction detected outside the ETDRS 7-field area.

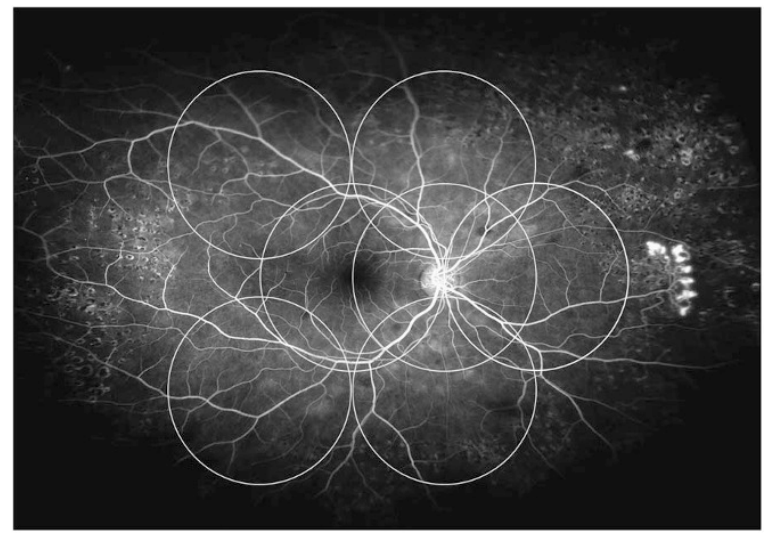

Figure 4 Retinal neovascularisation detected outside the ETDRS 7-field area.

\section{Discussion}

RV shows wide clinical variability, and may lead firstly to vascular wall inflammation and leakage of fluid, proteins, or cells anywhere within the retina; secondly, vascular occlusion with retinal haemorrhage, ischaemia, or infarction; and thirdly, retinal neovascularization with or without intravitreal haemorrhage. The identification of ischaemia in particular is an important milestone in management decision-making in RV. Vascular occlusion may be acute, typically symptomatic, and stepwise (for instance in Behçet's disease), but may be creeping and progressive, at worst ultimately leading to complete extinction of retinal vascular supply. The identification of ischaemia before substantial visual field loss is therefore important; both oral immunosuppression, and in some circumstances laser retinal ablation, are typically used in occlusive RV.

Widefield fundal imaging by definition gives greater coverage of potential fundal pathology, and the introduction of the Optos camera permitted up to $200^{\circ}$ visibility. ${ }^{3}$ Its superior coverage compared to standard $30^{\circ}$ views was rapidly apparent. ${ }^{4}$ Previous studies have demonstrated 76.4 sensitivity and $71.9 \%$ specificity for identifying retinal lesions. ${ }^{5}$

The increased visibility was shown to improve both detection and classification of diabetic retinopathy. ${ }^{6}$ In the field of uveitis, UWF FFA again captured more pathology from 52 eyes, ${ }^{7}$ but whether this changed clinical management was not clear. In Behçet's disease-associated $\mathrm{RV}$, Optos imaging detected additional pathology in $85 \%$ of 33 eyes. ${ }^{8}$ For RV generally, Optos UWF angiography together with pseudo-colour images was found in a study of 23 patients to alter management (potentially) in $51 \% .^{9}$

There are weaknesses in this study. First, its retrospective nature did not permit exact standardization of views including routine peripheral projections (but we would argue that the routine inclusion of these could only have increased, rather than decreased, the relative efficacy of UWF FFA); secondly, real ETDRS imaging was not performed, but an ETDRS transparent grid was superimposed and scaled onto an UWF FFA film to simulate the comparison. We would also argue here that 'real' ETDRS-style imaging, in positioning of fields, would be less consistent and would again probably have further decreased the 'real' diagnostic pickup of the ETDRS method; thirdly, only one unmasked observer (NPJ) made the comparisons, introducing the possibility of error and bias.

This study has shown a clear superiority of UWF FFA over standard 30-degree photography, in the identification of significant retinal vascular pathology in patients with RV. Optos UWF FFA imaging allowed easy and rapid identification of vascular changes. Pathology unseen by standard angiography fields was identified in more than half of all patients. This figure is smaller than that identified specifically for Behçet's disease, ${ }^{8}$ but this may reflect more diverse pathology. Changes to patient management were made in about one-third of patients 
following angiography, but importantly, one-fifth of patients underwent new treatment after new findings only detected by ultra-widefield imaging. Again, this figure is smaller than that identified by Leder et $a l^{9}$ for patients with RV, but arguably a $20 \%$ proportion as identified here, is ample justification for the use of this technique.

Ultra-widefield FFA has now become a standard diagnostic and monitoring tool in the Manchester Uveitis Clinic, for patients with inflammatory retinal vascular pathology. Its advantages in retinal coverage and reliability of image capture are clear, and it has been demonstrated to make a significant contribution to patient management.

\section{Summary}

What was known before

- Widefield photography permits instant recognition and orientation of retinal pathology.

What this study adds

- Widefield angiography is superior to montage or multiview standard angiography in identifying and treating pathology in vasculitis.

\section{Conflict of interest}

PES: Optos Plc. (consultancy, equipment, research funding, lecture fees, and travel expenses). The remaining authors declare no conflict of interest.

\section{Acknowledgements}

This research was partially facilitated by the NIHR/ Wellcome Trust Manchester CRF and the Greater Manchester Comprehensive Local Research Network by providing imaging equipment, clinical facilities, and clerical assistance.

\section{Disclaimer}

The authors alone are responsible for writing the article.

\section{References}

1 Jones NP. Manchester Uveitis Clinic: the first 3000 patients: 1. Ocul Immunol Inflamm 2015; 23: 118-126.

2 Staurenghi G, Viola F, Mainster MA, Graham RD, Harrington PG. Scanning laser ophthalmoscopy and angiography with a wide-field contact lens system. Arch Ophthalmol 2005; 123: 244-252.

3 Manivannan A, Plskova J, Farrow A, McKay S, Sharp PF, Forrester JV. Ultra-widefield fluorescein angiography of the ocular fundus. Am J Ophthalmol 2005; 140: 525-527.

4 Friberg TR, Gupta A, Yu J, Huang L, Suner I, Puliafito CA et al. Ultrawide angle fluorescein angiographic imaging: a comparison to conventional digital acquisition systems. Ophthalmic Surg Lasers Imaging 2008; 39: 304-311.

5 Cheng SC, Y M, Goldschmidt E, Swann PG, Ng LH, Lam CS. Use of the Optomap with lid retraction and its sensitivity and specificity. Clin Exp Optom 2008; 91(4): 373-378.

6 Wessel MM, Aaker GD, Parlitsas G, Cho M, D'Amico DJ, Kiss S. Ultra-widefield angiography improves the detection and classification of diabetic retinopathy. Retina 2012; 32: 785-791.

7 Nicholson BP, Nigam D, Miller D, Agron E, Dalal M, Jacobs-El $\mathrm{N}$ et al. Comparison of wide-field fluorescein angiography and nine-field montage angiography in uveitis. Am J Ophthalmol 2014; 157: 673-677.

8 Mesquida M, Llorenc V, Fonteneia JR, Navarro MJ, Adan A. Use of ultra-wide-field retinal imaging in the management of active Behçet retinal vasculitis. Retina 2014; 34: 2121-2127.

9 Leder HA, Campbell JP, Sepah YJ, Gan T, Dunn JP, Hatef E et al. Ultra-widefleld retinal imaging in the management of non-infectious retinal vasculitis. J Ophthalmic Inflamm Infect 2013; 3: 30. 